\title{
High sugar content of baby foods sold in Europe
}

\author{
Jayne Hutchinson ${ }^{1}$, Holly Rippin ${ }^{1}$, Diane Threapleton ${ }^{1}$, Jo Jewell ${ }^{2,3}$, Joao Breda ${ }^{4}$ and \\ Janet Cade \\ ${ }^{1}$ University of Leeds, Leeds, United Kingdom, \\ ${ }^{2}$ WHO Regional office for Europe, Copenhagen, Denmark, \\ ${ }^{3}$ UNICEF, New York, USA and \\ ${ }^{4}$ WHO European Office for the Prevention and Control of NCDs, Moscow, Russian Federation
}

\section{Abstract}

WHO guidelines on sugars strongly recommend reducing free sugar intake to below $10 \%$ of total energy, also their guidance on 'Ending the inappropriate promotion of foods for infants and young children (IYC)' recommends avoiding free sugars. Information on the sugar content of commercially available complementary foods (CACFs) for infants is limited, and the levels of added, free, and total sugar in baby foods is not fully addressed in existing guidelines. Up-to-date data is needed to inform recommendations for revising European regulations and guidelines. Front and back of pack information for baby foods from countries across the WHO European Region was collected. Mean sugar content per $100 \mathrm{~g}$ product and percentage energy from total sugar by food category were determined. The percentage of products containing added sugars by food category was calculated; as was the percentage of savoury meals containing pureed fruit, which is often used to sweeten products. The creation of the product categories was part of WHO work to develop a Nutrient Profile Model for CACFs marketed as suitable for IYC aged 6-36 months. A literature review of current issues also informed the process. 2642 CACFs from 10 countries were summarised; 768 products sold in the UK; over 200 each from Denmark (319), Spain (241), Italy (430) and Malta (243) and between 100-200 from Hungry, Norway, Portugal and Slovenia. About a third of energy in CACFs in these European countries came from total sugar and the mean contribution of total sugar for most food categories in the countries was higher than $10 \%$. The amount of added and free sugar content was not labelled. However, added sugars listed as ingredients were widespread across product categories, and the type varied across countries and products, though concentrated fruit juice was most common. Savoury meal purees did not contain added sugars except in UK and Malta; however fruit puree as an ingredient was found in 7\% of those examined across Europe and were most likely in UK savoury meals: in $15 \%$ on average and $41 \%$ of pureed meals with meat as the first named food. In conclusion, the sugar content of baby foods across Europe is high and updated regulations and reformulation are needed. Sugars can be restricted in a variety of ways, but first consultation with WHO Europe Member States is required to support the establishment of effective legal and policy measures to avoid inappropriate manufacture and selling of infant foods.

\section{Conflict of Interest}

The University of Leeds has established a spin out company myfood24, for our new on-line dietary assessment tool. Professor Janet Cade is a director and shareholder of myfood 24 . 